\title{
Symmetrical Periorbital Yellow Plaques in a Patient with Haematological Dyscrasia: A Quiz
}

Emanuele COZZANI ${ }^{1,2}$, Andrea MURACCHIOLI ${ }^{1,2 *}$, Giulio Fraternali ORCIONI ${ }^{3}$ and Aurora PARODI ${ }^{1,2}$

${ }^{1}$ Di.S.SAL Section of Dermatology, University of Genoa, ${ }^{2}$ Section of Dermatology, University of Genoa DiSSal, IRCCS Policlinic Hospital San Martino-IST, IT-16132 Genoa and ${ }^{3}$ Pathology Unit, ASO Santa Croce e Carle Cuneo, Italy. E-mail: andreamuracchioli@gmail.com

A 64-year-old woman was admitted to our department with lesions of the periorbital skin, which had appeared a few years previously and had increased progressively in size, limiting movements of her eyelids. The lesions had ulcerated repeatedly and become infected. Physical examination revealed hard, tender, and infiltrated bilateral brown-yellowish plaques of the periorbital area. The patient presented ectropion and ptosis with a limited range of eye excursions. No other lesions were observed on the skin surface (Fig. 1). Ophthalmological examination excluded keratitis, episcleritis and anterior uveitis. Visual acuity was preserved. The patient's medical history was remarkable for Cogan Syndrome with hypoacusia since 2013, lambda-monoclonal gammopathy of undetermined significance (MGUS, IgG type) with hypogammaglobulinemia diagnosed in 2015, and chronic lymphocytic leukaemia (LLC), diagnosed in September 2016 by bone marrow biopsy (RAI 0).

An incisional biopsy was performed for each ocular side, and histological examination showed a cellular inflammatory infiltrate of numerous foamy histiocytes and Touton-type giant cells accompanied by aggregations of

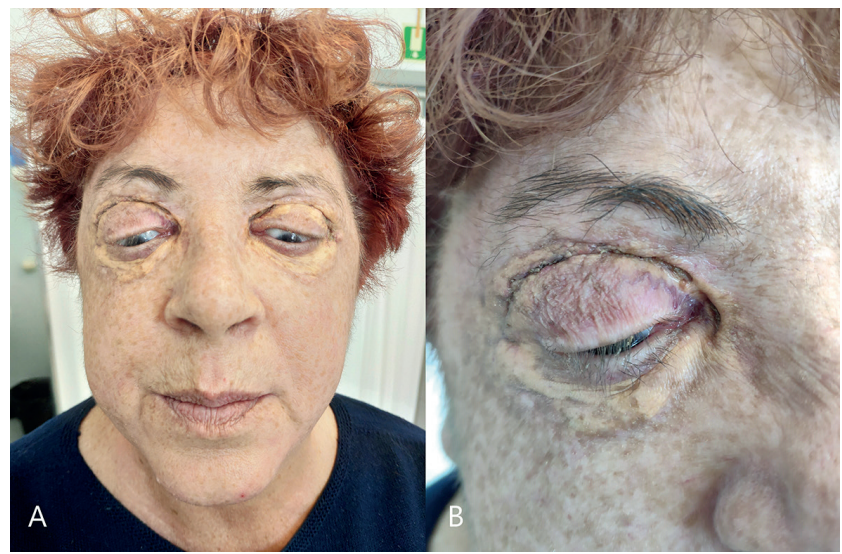

Fig. 1. (A) Clinical case presentation: infiltrated and elevated yellow, periocular plaques. The patient has important ptosis and difficulty in eyelid movement. (B) Detail of the periorbital lesion (right side). Permission was given by the patient to publish these photographs.

lymphocytes and plasma cells. S-100, CD1a, and CD 207 were negative (Fig. 2).

What is your diagnosis? See next page for answer.

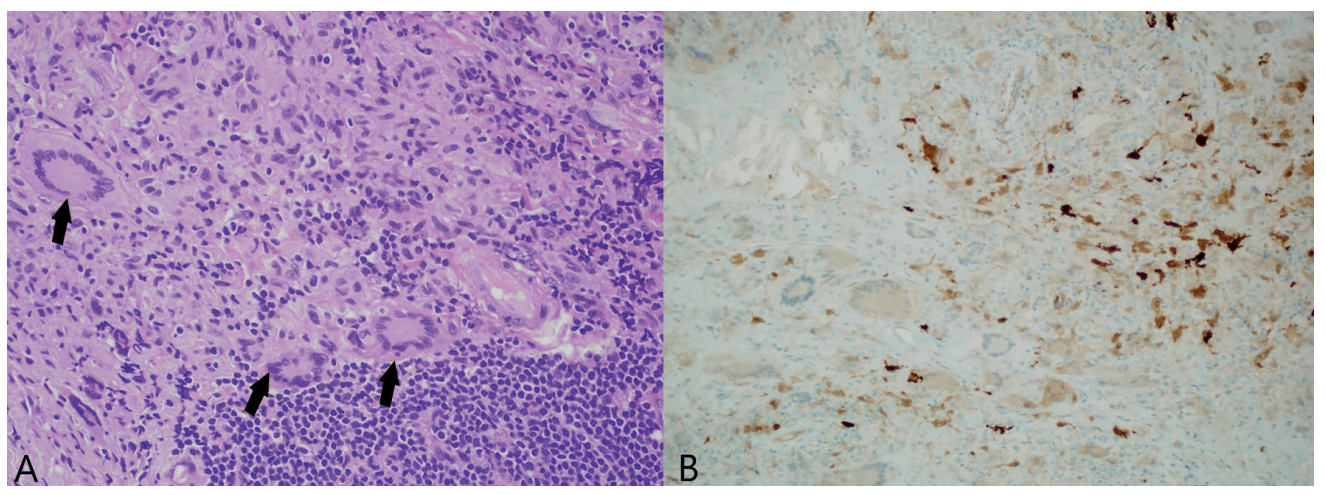

Fig. 2. (A) Histopathology of the periocular mass: mononucleated foamy histiocytes (xanthoma cells) infiltrating the lesion, with variable numbers of lymphocytes, plasma cells, and Touton giant cells (black arrows) (haematoxylin and eosin, $40 \times)$. (B) S-100 protein tested was negative $(S-100,20 \times)$. 


\section{ANSWERS TO QUIZ}

\section{Symmetrical Periorbital Yellow Plaques in a Patient with Haematological Dyscrasia: A Commentary}

Acta Derm Venereol 2021; 101: adv00612.

\section{Diagnosis: Necrobiotic xanthogranuloma}

Necrobiotic xanthogranuloma is a rare non-Langerhans histiocytosis ( $\mathrm{LCH})$ involving ocular and orbital tissue, characterized by the presence of yellow/purple-red coalescent nodule/plaque infiltrating the subcutaneous tissue. These lesions can ulcerate, necrotize, or fibrotize, resulting in alterations in eyelid motility or, in some cases, ocular motility. Extra-cephalic localizations, such as the trunk and the upper limbs, are rare, but have been reported (1). Haematological involvement is characteristic and variable, including lymphadenomegaly, IgG-type paraproteinemia, lymphoma, and myeloma $(1,2)$.

This rare idiopathic inflammatory disorders, together with the adult-onset xanthogranuloma (AOX), adult-onset asthma and periocular xanthogranuloma (AAPOX), and the Erdheim-Chester (ECD), is included in so-called adult-onset orbital xanthogranulomatous disease (AOXGD), a group of the non-Langerhans histiocytic proliferation of the orbit and periorbital region $(1,2)$. Although all these forms are characterized by similar cutaneous and histopathological features (histiocytosis of non-Langerhans mononuclear phagocytes, particularly foamy histiocytes and Touton multinucleated giant cells, negative for S100 and CD1a) the tissue infiltration, the systemic involvement and the prognosis of each are different $(2,3)$. The differential diagnosis between these 4 forms is performed by accurate clinical examination (assessing the severity of the skin extension, possible organ involvement), by haematological screening, and by imaging (computed tomography or magnetic resonance imaging to quantifies the degree of infiltration in the orbital cavity). AOX presents a minimum involvement of the periorbital area without systemic involvement and infiltration of the orbital cavity. AAPOX is characterized by systemic involvement, such as late-onset and drug-resistant asthma, lymphadenomegaly, and paraproteinaemia (monoclonal peak, mainly IgG-type). Large plaques or nodules in the periorbital region may be observed. Finally, ECD, the most severe form, is characterized by an aggressive involvement of the periorbital and orbital region (infiltration of the ocular cavity with alteration of extrinsic ocular motility and risk of vision loss), presence of xanthogranuloma in other body sites (neck, armpits, groin, and genitals) and a severe systemic involvement (cardio-pulmonary infiltration, aortic, retroperitoneal fibrosis, systemic bone sclerosis, diabetes insipidus).

Other more common non-histiocyte diseases, such as xanthelasma palpebrarum, skin sarcoidosis, granuloma annulare, and reaction to a foreign body, should be considered in the differential diagnosis $(4,5)$. The therapy, not yet standardized, consists of intralesional corticosteroid injections, immunosuppressants treatments (corticosteroids, cyclosporine, and methotrexate), and cytostatic drugs (vincristine, doxorubicin, and cyclophosphamide). Surgical debulking is a planned approach, especially for the AAOXP form, but not for NBX, due to a high recurrence rate (1). Radiotherapy has been reported to be effective in NXG (3). Regular follow-up is required to exclude the occurrence of new haematological dyscrasias. In the NXG subtype, haematological manifestations could appear within a median time range of 2.4-years, up to even 20 years, from the appearance of ocular-cutaneous manifestations $(1,5)$. The patient reported here initially received a systemic corticosteroid therapy (prednisone $1 \mathrm{mg} / \mathrm{kg}$ /daily) tapered and stopped after 4 months and replaced with the current $15 \mathrm{mg} /$ week methotrexate plus $5 \mathrm{mg} /$ week folic acid treatment. Partial resolution of the periorbital lesions was observed after 4 out of 10 fractions of radiotherapy (proposed protocol: 24 Gy in 10 fractions). Haematological dyscrasias have not worsened in some months of follow up.

The authors have no conflicts of interest to declare.

\section{REFERENCES}

1. Kerstetter J, Wang J. Adult orbital xanthogranulomatous disease: a review with emphasis on etiology, systemic associations, diagnostic tools, and treatment. Dermatol Clin 2015; 33: 457-463.

2. Guo J, Wang J. Adult orbital xanthogranulomatous disease: review of the literature. Arch Pathol Lab Med 2009; 133: 1994-1997.

3. Sivak-Callcott JA, Rootman J, Rasmussen SL, Nugent RA, White VA, Paridaens $D$, et al. Adult xanthogranulomatous disease of the orbit and ocular adnexa: new immunohistochemical findings and clinical review. $\mathrm{Br}$ J Ophthalmol 2006; 90: 602-608.

4. Pasadhika S, Rosenbaum JT. Ocular sarcoidosis. Clin Chest Med 2015; 36: 669-683

5. Ortiz Salvador JM, Subiabre Ferrer D, Pérez Ferriols A. Adult xanthogranulomatous disease of the orbit: clinical presentations, evaluation, and management. Actas Dermosifiliogr 2017; 108: 400-406. 